anti-PD-L1 checkpoint blockade, leading to eradication of large tumor. More importantly, HDAC8 and PD-L1 co-blockade resulted in long-term survival (more than 1 year) with the induction of $\mathrm{T}$ cell memory responses.

Conclusions Our finding delineates that selective chromatin modifications by HDAC8 can augment the therapeutic efficacy of PD-L1 blockade therapy to fully unleash $\mathrm{T}$ cell responses, leading to long-term remission of HCC. This study highlights a new epigenetic target for immune potentiation in HCC, providing a rational combinatorial epigenetic immunotherapy.

\section{IDDF2019-ABS-0326 EVALUATION OF THE EFFECTS OF THE HYDROALCOHOLIC EXTRACT OF LIV.52 DS ON PARACETAMOL INDUCED LIVER TOXICITY AND OXIDATIVE STRESS IN RATS}

Arun Gandhi*, Priya Kakkar. Faculty of Life Sciences, Maharshi Dayanand University, Rohtak, Haryana, India

\subsection{6/gutjnl-2019-IDDFabstracts.111}

Background Oxidative stress induced by toxicants is known to cause various complications in the liver. Herbal drug such as Liv.52 is found to have a hepatoprotective effect. However, the biochemical mechanism involved in the Liv.52 DS mediated protection against toxicity is not well elucidated using suitable in vivo models. Paracetamol causes oxidative stress and dysfunction of the liver.

This study was undertaken to evaluate the effects of the hydroalcoholic extract of Liv.52 DS on some biochemical and histopathological parameters of liver tissue in against paracetamol-induced hepatic damage in rats.

Methods Wistar rats were orally administered with $2 \mathrm{~g} / \mathrm{kg}$ body weight Paracetamol. Vehicle (distilled water) and silymarin $(50 \mathrm{mg} / \mathrm{kg}$ body weight) was used as the negative and positive control groups, respectively. Paracetamol-administered groups were treated with Liv 52 DS extract (100, 200, and $400 \mathrm{mg} / \mathrm{kg}$ ). After 15 days of treatment, the blood specimens and liver samples were examined. Alteration in the levels of biochemical markers of hepatic damage like AST, ALT, ALP and lipid peroxides were tested, and phytochemical tests were also performed.

Results In Paracetamol-treated group, the levels of serum urea, high-density lipoprotein (HDL), and liver superoxide dismutase (SOD), catalase (CAT), and vitamin C significantly decreased $(\mathrm{p}<0.05)$ compared to control. Also, in this group, serum triglyceride (TG), total cholesterol (TC), very low-density lipoprotein cholesterol (VLDL), protein carbonyl (PC), malondialdehyde, tumor necrosis factor- $\alpha$ (TNF- $\alpha$ ), and TNF$\alpha$ gene expression significantly increased $(\mathrm{p}<0.05)$ as compared to the control (vehicle-treated rats). Treatment with Liv. 52 DS extract in a significant increase $(\mathrm{p}<0.05)$ in CAT, SOD, vitamin $C$, HDL and a significant decrease $(p<0.05)$ in the level of urea, MDA, PC, TG, TC, VLDL, TNF- $\alpha$ protein, and the gene expression of TNF- $\alpha$ compared with the test without treatment group. Histopathological evidence demonstrated that treatment with Liv.52 DS extract could decrease liver lymphocyte infiltration.

Conclusions The present study suggests that Liv. 52 DS extract possesses hepatoprotective activity. It could be an effective and promising preventive agent against Paracetamol-induced hepatotoxicity.

\section{IDDF2019-ABS-0334}

CLINICAL ESCHERICHIA COLI NF73-1

ISOLATED FROM A PATIENT WITH NONALCOHOLIC STEATOHEPATITIS INDUCES LIVER INJURY THROUGH IMPAIRING INTESTINAL BARRIER FUNCTION AND INDUCING INFLAMMATORY RESPONSES OF HEPATOCYTES

Yang Song*, Zhe Wu, Jun Xu, Yujing Chi, Yifan Zhang, Yulan Liu. Peking University People's Hospital, China

\subsection{6/gutjnl-2019-IDDFabstracts. 112}

Background Gut microbiota plays critical roles in nonalcoholic fatty liver disease (NAFLD). We have previously isolated and identified one clinical Escherichia coli (E. coli) strain from the intestinal mucosa of a nonalcoholic steatohepatitis (NASH) patient, and named it as E. coli NF73-1. Our aim is to investigate the role of E. coli NF73-1 in the development of NAFLD in a high-fat diet (HFD) mice.

Methods Conventional (CV) mice, plus mice treated with antibiotics $(\mathrm{AB})$ to deplete gut microbiota, were fed with HFD for 12 weeks. At the $10^{\text {th }}$ week, mice were treated daily with oral gavage of LB, live-NF73-1, or pasteurized-NF73-1 (pas-NF73-1) for 2 weeks. $\mathrm{AB}$ mice were treated in drinking water containing $1 \mathrm{~g} / \mathrm{L}$ ampicillin, $500 \mathrm{mg} / \mathrm{L}$ vancomycin, $1 \mathrm{~g} / \mathrm{L}$ neomycin, and 1 $\mathrm{g} / \mathrm{L}$ metronidazole for 4 weeks, starting at $6^{\text {th }}$ week. In vitro bacterial translocation and transepithelial permeability assay was performed. Primary hepatocytes from NAFLD mice were cocultured with NF73-1 to evaluate inflammatory responses.

Results Live-NF73-1 group developed severer liver pathology than $\mathrm{LB}$ and pas-NF73-1 groups in both $\mathrm{CV}$ and $\mathrm{AB}$ mice, verified by increased NAFLD activity (NAS) score. Besides, intestinal permeability was higher in live-NF73-1 group than that in LB and pas-NF73-1 groups of both $\mathrm{CV}$ and $\mathrm{AB}$ mice, supported by decreased expression of ZO-1 and Occludin in the colon. In vitro bacterial translocation and transepithelial permeability assay indicated that HT-29 cells treated with live-NF73-1 developed a higher concentration of translocated bacteria and FITC fluorescein than LB and pas-NF73-1 groups. Interestingly, live-NF73-1 decreased mRNA levels of ZO-1, Occludin and Claudin2 in Caco 2 cells, and downregulated mRNA expression of Claudin2 and E-cadherin in HT-29 cells compared with LB and pasNF73-1 groups. NF73-1 also induced inflammatory responses of primary hepatocytes, supported by increased IL-6 expression.

Conclusions Clinical E. coli NF73-1 aggravates liver injury in NAFLD mice, through impaired intestinal integrity and inflammatory responses in hepatocytes. These findings provide new insights on management using specific bacterial strain.

\section{Clinical Gastroenterology}

\section{IDDF2019-ABS-0013 CELIAC CRISIS IN AN ADULT TYPE 1 DIABETES MELLITUS PATIENT PRESENTING WITH DIARRHEA, WEIGHT LOSS AND HYPOGLYCEMIC ATTACKS- A RARE ENTITY}

Shivam Shivam*, Pratibha Nadig, Vijaya Sarathi, Manohar KN. Vydehi Institute of Medical Sciences and Research Centre, India

10.1136/gutjnl-2019-IDDFabstracts. 113

Background Type 1 diabetes mellitus (T1DM) is an autoimmune disease, characterized by loss of insulin-producing beta 